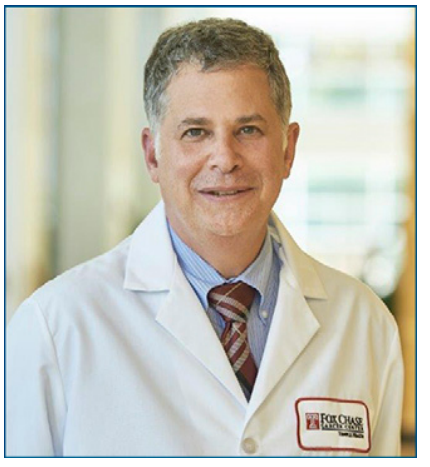

MARTIN J. EDELMAN, MD

Martin J. Edelman, MD, is the G. Morris Dorrance Professor and Chair of the Department of Hematology/Oncology and Deputy Director for Clinical Research at Fox Chase Cancer Center. His research focus has been on lung and thoracic malignancy with a focus on investigational therapeutics, but has also included research into outcomes and supportive oncology. He has been active in the U.S. National Clinical Trials Network and a member of the ECOG Lung Cancer Committee and was previously with CALGB and SWOG. He is a member of the Thoracic Malignancies Steering Committee of the $\mathrm{NCl}$. He has also served as the Chair of the Integration Panel for the DOD Congressionally Mandated Research Program for lung cancer, a member of the NIH subcommittee $\mathrm{H}$, the ASCO program, education and governmental affairs committees and several $\mathrm{NIH}$ study sections. He is past Chair of the Ethics Committee and the Career Development Committee, a member of the Communications Committee of the IASLC, and deputy editor of Lung Cancer. He is currently Chair of the Scientific Advisory Board for the Lung Cancer Foundation of America. He has published over 190 peer reviewed papers.

doi: $10.6004 /$ jnccn.2020.7588

The ideas and viewpoints expressed in this commentary are those of the author and do not necessarily represent any policy, position, or program of NCCN.

\section{Clinical Trials in the Age of Pandemics}

\author{
Martin J. Edelman, MD*; Crystal S. Denlinger, MD*; \\ Eric A. Ross, PhD, ScM; and Margaret von Mehren, MD
}

C linical trials play a unique role in the treatment of patients with cancer. Although only a small percentage of patients actually enter studies, enrollment onto a trial is considered a "standard of care" for virtually all patients with advanced or locally advanced cancer. Even for diseases for which therapeutics have achieved high cure rates, there are trials evaluating strategies to minimize duration and toxicity of treatment.

The effort required to formulate, activate, accrue, and analyze trials is considerable; even small trials require substantial resources. Large, definitive trials may consume hundreds of person-hours, span the globe, and cost in excess of $\$ 100$ million. Before the current pandemic, experts recognized that the existing system imposed an undue regulatory and administrative burden on investigators and had arbitrary entry criteria that restricted enrollment. ${ }^{1,2}$

The COVID-19 pandemic has disrupted all aspects of social, work, and economic life throughout the world. ${ }^{3,4}$ Although completion of clinical trials in cancer is certainly not the most pressing issue at this time, it is important to patients, physicians, and sponsors. The substantial morbidity and mortality for patients with cancer is not going to go away. Furthermore, patients with cancer with COVID-19 infection are at high risk for adverse outcomes. ${ }^{5} \mathrm{~A}$ marked decline in screening and accrual to trials within the National Clinical Trials Network has been noted. ${ }^{6}$ Regulations governing clinical trials allow for the occurrence of study modifications to be implemented before Institutional Review Board (IRB) approval without adverse action only when it is necessary to eliminate apparent immediate hazards to the subject. ${ }^{7}$ This allows investigators to modify protocol-required visits or make other protocol changes if it is in the best interests of the subject. These deviations must be reported to the IRB of record within 5 days.

Agencies including the $\mathrm{NCl}$ and the FDA have issued guidance regarding management of patients on clinical trials during the pandemic. ${ }^{8-10}$ As previously noted, modifications can be made in the interest of patient safety without adverse action. Additionally, if modifications to protocol assessment schedules can be proactively identified to minimize risk to subjects, these can be submitted to the IRB of record as a planned deviation before implementation, with short turnaround times from submission to IRB approval to optimize subjects' outcomes. The $\mathrm{NCl}$ guidance contains procedures for forwarding some investigational agents directly to patients.

However, the guidance is nonbinding on industry, may result in further confusion, and does not address the next disruptive event. The $\mathrm{NCl}$ guidance applies only to $\mathrm{NCl}$-sponsored research, and the FDA guidance can be interpreted variably by sponsors. Critically, substantial burden is placed on investigators and their institutions in terms of absorbing the risk of being deemed noncompliant with the IRB- and sponsor-approved protocol. Such risk could include significant financial liability if sponsors penalize institutions for such "noncompliance" as well as the potential for adverse action by the FDA (eg, issuance of a form 483).

It is important to recognize that, regardless of when the current pandemic ends, similar disruptive events from disease and/or natural or manmade disasters will inevitably occur in the future. Planning for such events is a necessity. The following are a series of specific proposals for emergency trial guidelines that we hope will start discussions and policies in anticipation of future disruptive events to mitigate their impact on clinical research efforts in oncology. 
1. All studies should have a clear set of contingencies regarding the ability to delegate study procedures to other practitioners outside of the investigator team or through remote means as part of the initial protocol. Guidelines for activating these contingencies should be delineated within the protocol document and be consistent across industry sponsors. This will eliminate the need for amendments requiring IRB approvals or for the generation of study deviation reports.

2. To activate contingencies in the setting of a disruptive event, site investigators should ascertain that continuing individual trial participants on study therapy is in the patient's best interest and document that contingency plan activation is being conducted for the safety and best interest of the participants.

3. In circumstances in which patients are traveling to investigative sites or when it is considered in the best interest of the patient to avoid the investigative site, contingency plans should include a clear mechanism and oversight plan for "deputizing" local healthcare professionals to perform examinations and toxicity assessments for study participants. This could include permitting a participant's referring oncology or primary care provider to conduct clinical assessments. Many assessment visits require straightforward history and examination. It would not be difficult to have local healthcare providers complete basic paperwork (eg, disclosure, FDA form 1572, clinical research training) remotely and review an activations slide set to acquaint themselves with basic information regarding a specific trial. It is routine at our center for the slides from site initiation visits to be distributed to all investigators, including those who may not have been present at the actual meeting. A signed document indicating review of these slides, particularly those dealing with assessment of toxicity, could be submitted remotely. Site investigators with a large network of outside referring physicians should engage participants' local healthcare teams early in treatment to ensure a smooth continuation of assessments and care, should the need arise. An alternative to this approach is the use of telehealth visits for many of the "routine" visits that are mandated as part of trials. We have used this approach for many study visits during the current pandemic. Although such visits do not allow for physical examination assessment, they are appropriate for determining symptomatic toxicities. ${ }^{11}$

4. Results of routine laboratory studies (eg, CBC counts, chemistries) from facilities other than the study institution should be accepted as source data, provided those laboratories are CLIA-certified. In addition, if possible, such laboratories should also be able to draw and ship study-related pharmacokinetic and other samples. Third-party home phlebotomy and visiting nurse services could be engaged to allow standard sample collection for needed studies without participants having to travel to distant study centers.

5. Provision of drug directly to participants for oral agents or to suitable practitioners (eg, community oncologists) for oral and parenteral agents should be allowed. Procedures for drug delivery through commercial carriers should be developed in the setting of travel restrictions. It is very common for community physicians to participate in some aspects of research through the National Clinical Trials Network mechanism or through industry trials, and therefore they are frequently familiar with the basic tenets of drug accountability. Again, this could be accomplished through engagement with participants' local oncology providers.

6. Operating procedures for obtaining participants' informed consent remotely for protocol amendments describing new risks of procedures requiring

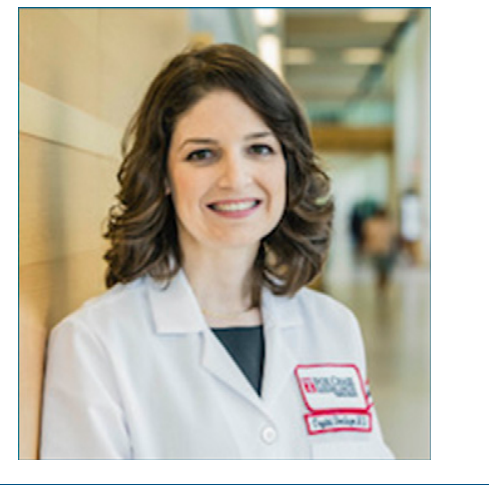

CRYSTAL S. DENLINGER, MD

Crystal S. Denlinger, MD, is an Associate Professor and medical oncologist with a clinical focus in gastrointestinal malignancies and research interests in drug development and cancer survivorship in upper $\mathrm{Gl}$ malignancies. She serves as Chief of GI Medical Oncology, Deputy Director of the Phase I Program, and Director of the Survivorship Program at Fox Chase Cancer Center. Dr. Denlinger chairs the NCCN Guidelines Panel for Survivorship, is an active member of the Esophagogastric Cancer Panel, and has collaborated with a number of gastrointestinal cancer panels on developing principles of survivorship care. She is a member of ASCO's Survivorship Guidelines Advisory Group and active in multiple professional and patient advocacy organizations' efforts targeting the survivorship experience. In addition, she is an active clinical investigator for both industryand cooperative group-sponsored trials in both gastrointestinal cancers and phase I trials. She leads Fox Chase Cancer Center's gastrointestinal cancers clinical trials programs and oversees survivorship programming efforts across the institution. 


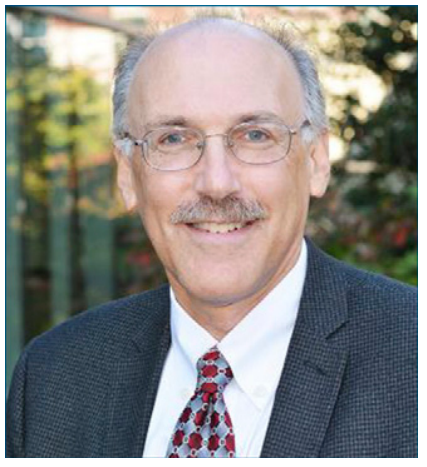

ERIC A. ROSS, PhD, ScM

Eric A. Ross, PhD, ScM, is Assistant Vice President of Biometrics and Information Sciences and Research Professor at Fox Chase Cancer Center. He is also the Director of the Biostatistics and Bioinformatics Facility and the Population Studies Facility. His research has focused on the innovative use of quantitative methods and information technology in cancer research, including creation of efficient clinical trial designs, high throughput data analysis, and facilitating biomedical research through data warehousing. Dr. Ross is an associate editor for statistics for Annals of Internal Medicine and member of the ECOG-ACRIN Prevention Committee. He has published over 160 peer-reviewed manuscripts. repeat consent should be outlined. These may include the use of video platforms, audio recordings, and other electronic media to send and receive verbal and written informed consent for protocol changes, but they would not be applicable to new participant consents to initiate study treatment.

7. Mechanisms and procedures for urgent transfers of care from one study center to another should be outlined. Such transfers should allow for participant identification numbers to travel with the participant to the new site to allow for continuation of care while the formal transfer of care and data are accomplished. This will allow study-related data to be collected and assigned to the correct participant while minimizing interruptions in treatment cycles. The transfer of care should be able to be accomplished regardless of the status of data submission or query resolution at the initiating site.

8. Mechanisms to allow for remote monitoring of trials should be in place prior to activation. This would include methods of accessing electronic medical records and other relevant data. Procedures for discussions with investigators and study staff by telephone or other methods not requiring direct contact should be delineated.

9. Statistical methods to evaluate the results of trials that use such contingency plans should be part of the initial study document. For example, sensitivity analyses could be planned to explore whether the cohort of participants treated under emergency contingency guidelines may bias trial conclusions. Furthermore, it is quite possible that patients who are participating in trials may succumb or become nonevaluable due to the epidemic disease or other event. The statistical plan should describe whether these patients will be replaced, supplemented, or excluded.

The current era of oncology clinical trials holds immense promise for effective therapies to drive continued reductions in cancer-related morbidity and mortality. The COVID-19 pandemic has disrupted clinical research of promising therapies in ways no one had previously considered. As investigators, we do not want our progress derailed by failure to plan for inevitable unforeseen disasters, natural or manmade. Contingency approaches such as those detailed previously should be put in place as soon as possible for existing trials, and incorporated as a matter of course into future trials prior to activation. It is already clear that clinical trials in oncology can proceed, even in the most severely impacted areas. The phase I unit at the Department of the European Institute of Oncology in Milan has shown that measures such as those described previously can allow for continued accrual to even the most complex studies. ${ }^{12}$ Planning for the unforeseen now ensures that we will be able to continue to advance the field of oncology while simultaneously confronting other challenges to human health in the future.

*These authors contributed equally to the manuscript.

Disclosures: Dr. Edelman has disclosed that he has served on the data safety and monitoring board for Astra-Zeneca, GlaxoSmithKline, and Takeda); has served on scientific advisory boards for Windmil, and Biomarker Strategies; has received research support from Windmil, GlaxoSmithKline, Merck, Nektar, Revolutions Medicine, and Apexigen. Dr. Denlinger has disclosed that she has served as a scientific advisor for Exelixis, Astellas, Bayer, Bristol Myer Squibb, and Taiho; and has received insitutional research support from Bristol Myer Squibb, Merrimack, Astra Zeneca, Eli Lilly, Roche, Amgen, Sanofi Aventis, BeiGene, Lycera, Macrogenics, Agios Pharmaceuticals, and Zymeworks. Dr. Ross has disclosed that he has a patent pending on screening for chemotherapy responsiveness and owns stock from Protor \& Gamble. Dr. von Mehren has disclosed that she has served on scientific advisory boards for Deciphera, Blueprint, and Exelis; has received institutional research support from Deciphera, Blueprint, Arog Pharmaceuticals, Gardalis, Springworks, Novartis, and ASCO; and has received honorarium from Pfizer and ASCO.

Correspondence: Martin J. Edelman, MD, Fox Chase Cancer Center, 333 Cottman Avenue, Philadelphia, PA 191111. Email: Martin.Edelman@fccc.edu 


\section{References}

1. Vose JM, Levit LA, Hurley $\mathrm{P}$, et al. Addressing administrative and regulatory burden in cancer clinical trials: summary of a stakeholder survey and workshop hosted by the American Society of Clinical Oncology and the Association of American Cancer Institutes. J Clin Oncol 2016;34:3796-3802.

2. Kim ES, Bruinooge $S S$, Roberts $S$, et al. Broadening eligibility criteria to make clinical trials more representative: American Society of Clinical Oncology and Friends of Cancer Research Joint Research Statement. J Clin Oncol 2017;35:3737-3744.

3. Guan WJ, Ni ZY, Hu Y, et al. Clinical characteristics of coronavirus disease 2019 in China. N Engl J Med 2020;382:1708-1720

4. Fauci AS, Lane HC, Redfield RR. Covid-19 - navigating the uncharted. N Engl J Med 2020;382: 1268-1269.

5. Liang W, Guan W, Chen R, et al. Cancer patients in SARS-CoV-2 infection: a nationwide analysis in China. Lancet Oncol 2020;21:335-337.

6. Ong MBH. Doroshow: $\mathrm{NCl}$ to accrue patients for COVID-19 longitudinal cohort: ongoing trials see significant decrease in accrual. The Cancer Letter. Accessed March 23, 2020. Available at: https:// cancerletter.com/articles/20200417_2/

7. Code of Federal Regulation $\$ 46.108$ (a)(3)(iii) and 21 CFR 56.108(a)(4).

8. Mooney M, McCaskill-Stevens W. Interim guidance for patients on clinical trials supported by the $\mathrm{NCl}$ Cancer Therapy Evaluation Program and the NCl Community Oncology Research Program (NCORP). Accessed March 23, 2020. Available at: https://ctep.cancer.gov/content/docs/Memorandum_on Interim_Guidance_for_Clinical_Trial_Activities_Affected_by_the_Novel_Coronavirus-3-13-2020.pdf

9. FDA guidance on conduct of clinical trials of medical products during the COVID-19 pandemic. Accessed July 10, 2020. Available at: https://www.fda.gov/media/136238/download

10 Mooney M, McCaskill-Stevens W. Additional guidance regarding alternative procedures for clinical trials supported by the $\mathrm{NCl}$ Cancer Therapy Evaluation Program (CTEP) and $\mathrm{NCl}$ Community Oncology Research Program (NCORP) affected by the spread of the novel coronavirus. Accessed March 23, 2020. Available at: https://ncorp.cancer.gov/d/Memorandum-on-Additional-Guidance-forClinical-Trial-Activities-Affected-by-the-Novel-Coronavirus_3-23-2020.pdf

11. Hollander JE, Carr BG. Virtually perfect? Telemedicine for Covid-19. N Engl J Med 2020;382: 1679-1681.

12. Tarantino $P$, Trapani $D$, Curigliano $G$. Conducting phase 1 cancer clinical trials during the severe acute respiratory syndrome coronavirus 2 (SARS-CoV-2)-related disease pandemic. Eur J Cancer 2020;132: 8-10.

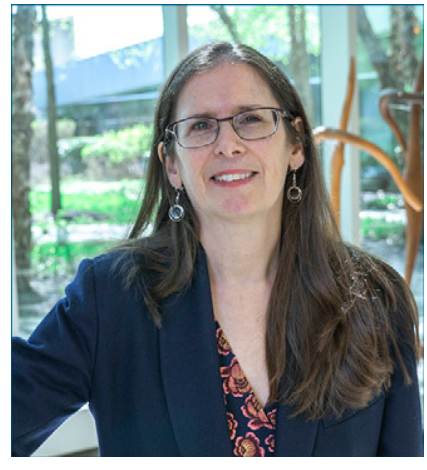

MARGARET VON MEHREN, MD

Margaret von Mehren, MD, Professor in the Department of Hematology Oncology at Fox Chase Cancer Center, serves as the Chief for Sarcoma Oncology. She was appointed the Physician Director of the Office of Clinical Research in 2011 and the Associate Director for Clinical Research in 2014. She has lead numerous trials testing novel therapeutics in gastrointestinal stromal tumors and soft tissue sarcomas. 